\title{
Dynamics of polycarbonate far below the glass transition temperature: A positron annihilation lifetime study
}

\author{
D. Cangialosi ${ }^{1,2}$ M. Wübbenhorst ${ }^{1}$ H. Schut, ${ }^{3}$ A. van Veen,${ }^{3}$ and S. J. Picken ${ }^{1,2}$ \\ ${ }^{1}$ Department of Polymer Materials and Engineering, Delft University of Technology, Julianalaan 136, 2628 BL, Delft, The Netherlands \\ ${ }^{2}$ Dutch Polymer Institute, P. O. Box 902, 5600 AX Eindhoven, The Netherlands \\ ${ }^{3}$ Interfaculty Reactor Institute, Delft University of Technology, Mekelweg 15, $2629 \mathrm{JB}$, Delft, The Netherlands
}

(Received 31 July 2003; published 26 April 2004)

\begin{abstract}
In this study, we have extended the knowledge of the dynamics of polycarbonate (PC) far below $T_{g}$ by means of positron annihilation lifetime spectroscopy (PALS), which is able to detect the isothermal relaxation of the free volume after rejuvenation above $T_{g}$. The free volume relaxation patterns at different temperatures below $T_{g}$ were modeled according to a phenomenological model presented by Struik to obtain the equilibrium relaxation times of the process at each aging temperature. The dynamics of supercooled liquids above the glass transition temperature $\left(T_{g}\right)$ is usually described by the Williams-Landel-Ferry (WLF) or Vogel-FulcherTammann (VFT) equation. Recently, deviations from this behavior were found below $T_{g}$. In contrast, an Arrhenius-type temperature dependence was found. Our results suggest that, far below $T_{g}$, two possible scenarios can hold: (a) the relaxation process of PC is dominated by the $\beta$ process, though the fast modes of the $\alpha$ process still manifest even at the lowest investigated temperature; (b) an Arrhenius-type process, deriving from the coupling of few $\beta$ events, drives aging far below $T_{g}$. Finally, the aging process was related to a diffusion mechanism and a coupling parameter was introduced as a measure of the efficiency of the $\beta$ process in producing appreciable changes in the density. The magnitude of the coupling parameter suggests a weak coupling between the $\beta$ relaxation and the physical aging process.
\end{abstract}

DOI: 10.1103/PhysRevB.69.134206

PACS number(s): 61.43.Fs, 61.20.Lc, 82.35.Lr

\section{INTRODUCTION}

Many liquids, like low-molecular-weight organic compounds, metals and polymers, may avoid crystallization when cooled down from the melt. Instead for kinetic reasons they remain in a quasiequilibrium liquid state. ${ }^{1}$ These materials are generally known as glass formers, since a further temperature reduction causes the formation of a "frozen-in" state where relaxation phenomena are not visible on the time scale of feasible experiments. Depending on temperature, the equilibrium state may not be reached even over a time scale of years. The nonequilibrium nature of these phenomena makes the study of the physics of the glassy state somewhat complicated. In fact, the dynamics of supercooled liquids is only well understood at temperatures close to the glass transition temperature $T_{g}$. In particular, the relaxation exhibits a deviation from simple Arrhenius behavior above $T_{g}$, and this is usually described by the so-called Vogel-FulcherTammann $^{2-4}$ (VFT) or the Williams-Landel-Ferry ${ }^{5}$ (WLF) equation. Both of these predict a large temperature dependence of the relaxation time. For example, according to the VFT equation, the relaxation time is written as follows:

$$
\tau=\tau_{0} \exp \left(\frac{E_{\mathrm{V}}}{k_{B}\left(T-T_{\mathrm{V}}\right)}\right)
$$

where $\tau_{0}$ is a prefactor, $E_{\mathrm{V}}$ is the so-called Vogel activation energy that is a material parameter, and $T_{\mathrm{V}}$ is the temperature at which the relaxation time diverges to infinity.

Supercooled liquids are commonly divided in two groups, strong and fragile glass formers, depending on the strength of the relaxation dependence on temperature. ${ }^{6,7}$ The former group is composed of supercooled liquids where the relaxation time has a weak dependence on temperature (up to the Arrhenius limit, like in silica), while the latter are supercooled liquids that undergo a progressive increase of the relaxation time when $T_{g}$ is approached from above. As a consequence, this second group of materials has a $T_{\mathrm{V}}$ value that is well above absolute zero. Though the strong-fragile classification has nothing to do with the mechanical properties, the degree of curvature of the relaxation pattern can be interpreted in terms of intermolecular cooperativity, which also shows a strong correlation with the Kohlrausch-WilliamsWatt (KWW) exponent ${ }^{8}$ of the stretched exponential. ${ }^{9-11}$

The extrapolation of the relaxation times according to the VFT law leads to an infinite mean relaxation time to occur at $T_{\mathrm{V}}$. However, the fast processes related to the $\alpha$ relaxation can be present even below $T_{\mathrm{V}}$, at least in the hypothesis of a heterogeneous nature of the glassy state. ${ }^{12}$ Moreover, polycarbonate (PC), which has a $T_{\mathrm{V}}$ of $98^{\circ} \mathrm{C}$ as evaluated by extrapolating dielectric data, ${ }^{13}$ still undergoes structural relaxation at room temperature as shown in the past by several aging experiments. ${ }^{14-17}$ The existence of $T_{\mathrm{V}}$, where the relaxation time diverges to infinity, was explained from free volume theories, ${ }^{5,18}$ and by the Adams-Gibbs relation between viscosity and configurational entropy. ${ }^{19}$ In this context pioneering work by Nozaki and Mashimo ${ }^{20}$ investigated the possibility that the VFT behavior is no longer valid below $T_{g}$ while performing dielectric studies on poly(vinyl acetate). More recently, theoretical results ${ }^{21}$ and in particular aging experiments below $T_{g}$, which revealed to be a useful tool to study the dynamics in the glassy state, ${ }^{22,23}$ also suggested that just below $T_{g}$ a deviation from the VFT behavior occurs. In particular, the volume and enthalpy recovery experiments 
on polystyrene (PS) of Simon et al. ${ }^{22}$ and the stress relaxation experiments on PC of O'Connell and McKenna ${ }^{23}$ showed that as $T_{g}$ (defined as the temperature at which the relaxation time is $100 \mathrm{~s}$ ) is approached and passed from above, the shift factor $a_{\mathrm{T}}$, defined by the superposition of relaxation curves at different temperatures, seems to show an Arrhenius-type behavior. Although the available temperature range below $T_{g}$ is too limited to clearly demonstrate a transition to an Arrhenius behavior, it is evident from these experiments that the equilibrium relaxation times below $T_{g}$ are smaller than predicted by the VFT model. The studies of Alegria et al. ${ }^{24}$ by means of time domain dielectric spectroscopy and enthalpy relaxation below $T_{g}$ and, very recently, the studies of Thurau and Ediger ${ }^{25}$ on PC by means of rotational and translational diffusion of probe molecules, indicate a similar trend. Finally, the time domain Brillouin spectroscopy experiments on diglycidyl ether of bisphenol A (DGEBA) of Kruger et al. ${ }^{26}$ indicate not only deviation from the VFT behavior, but also the disappearance of the $\alpha$ process below a transition temperature.

The present work aims to extend the measurements of the temperature dependence of the relaxation time to temperatures far below $T_{g}$. At present, the role of the different relaxation processes occurring far below $T_{g}$ is still unclear in the physical aging process. The main focus is to elucidate the role of the primary $\alpha$ and secondary $\beta$ relaxation processes. To achieve this aim, the physical aging of PC that occurs after rapid cooling from the melt was studied to investigate the temperature dependence of the relaxation time of the equilibrium glassy state. To describe our results we use a phenomenological model presented by Struik, ${ }^{27}$ which is based on the idea that aging is a self-retarding process where the relaxation time rapidly increases due to a decrease in the excess free volume. This model is expressed by the two following equations:

$$
\begin{gathered}
\frac{d v_{f}}{d t}=-\frac{v_{f}-v_{f \infty}}{\tau\left(T, v_{f}\right)}, \\
\ln \tau=\ln \tau_{0}+\frac{E_{a}}{k_{B} T}-\gamma\left(v_{f}-v_{f \infty}\right),
\end{gathered}
$$

where $E_{a}$ is the activation energy, $\ln \tau_{0}$ is a preexponential factor, $v_{f}-v_{f \infty}$ is the difference between the actual free volume and the equilibrium free volume (the excess free volume), and $\gamma$ is a material constant independent of temperature. This model describes the relaxation time as a function of both the temperature and the distance from the equilibrium glass, i.e., the structure of the glass, and explicitly makes use of the distance from equilibrium.

In the present study, free volume relaxation of PC is investigated by means of positron annihilation lifetime spectroscopy (PALS). In our previous publication, ${ }^{14}$ we have shown the possibility of studying PC physical aging by PALS. In this technique, positrons coming from a radioactive source of ${ }^{22} \mathrm{Na}$ are injected into the polymer sample where they annihilate. At least three different pathways for positron annihilation with an electron occur in condensed matter: the first is the direct annihilation process with an electron where the typical lifetime is between 300 and 500 ps; the second and the third processes occur after the formation of a positron-electron bound state, which, depending on the relative spin of the two particles, is called parapositronium ( $p$-Ps anti-parallel spin) and orthopositronium ( $o$-Ps parallel spin). ${ }^{28}$ In vacuum, $p$-Ps has a lifetime of about $125 \mathrm{ps}$ and $o$-Ps of about 142 ns but in matter the latter lifetime is shortened to $1-5 \mathrm{~ns}$ depending on the free volume properties of the sample. In particular, the $o$-Ps lifetime is related to the size of the free volume cavity through the following semiempirical equation. ${ }^{29}$

$$
\tau_{o-\mathrm{Ps}}=\frac{1}{2}\left[1-\frac{R}{R+\Delta R}+\frac{1}{2 \pi} \sin \left(\frac{2 \pi R}{R+\Delta R}\right)\right]^{-1},
$$

where the lifetime is expressed in nanoseconds and $\Delta R$ is the electron layer thickness $(1.656 \AA)$.

The intensity of $o$-Ps $\left(I_{o-\mathrm{Ps}}\right)$ is proportional to the probability of formation of this species. Since $o$-Ps preferably localizes in region of low electron density ${ }^{28}$ the probability of its formation is generally related to the number of cavities present in the system. ${ }^{30}$ In this manner, the total fractional free volume can be evaluated from the following equation:

$$
v f=A \frac{4}{3} \pi R^{3} I_{o-\mathrm{Ps}},
$$

where $A$ is a constant taken from literature data.

In the past, several studies concerning aging of PC and other amorphous polymers using PALS have been presented. ${ }^{31,32}$ Although these publications deserve attention since they introduced a new approach for studying aging of glassy amorphous polymers, the $I_{o \text {-Ps }}$ decrease curves that were reported seem to be biased by the radiation effects caused by prolonged exposure to the positron source. As a matter of fact, the relaxation times obtained in these aging studies are much too short to be attributed to molecular rearrangement. This was also noted by Hutchinson in a review on physical aging. ${ }^{33}$ As a consequence the activation energy for the physical aging process obtained by Hill et al. ${ }^{31}$ is too low to be fully attributed to aging.. The main reason for this is probably that a single sample was used for the entire aging study, which ultimately leads to a modification of the structure of the sample in terms of the internal electric field and the free radical concentration. Extensive studies of these radiation effects, which interfere with the probability of $o$-Ps formation, have been reported over the last ten years. ${ }^{34,35}$

In the present study, the free-volume reduction of $\mathrm{PC}$ is investigated by means of PALS at different temperatures below $T_{g}$. In order to avoid artefacts resulting from charging effects during the positron measurement, the number of counts collected for each sample was $2 \times 10^{6}$, which is low enough to avoid any significant charging during the measurement. ${ }^{34}$

This article introduces new aging experiments by means of PALS at 50 and $65^{\circ} \mathrm{C}$ that supplement results we published recently. ${ }^{14}$ Apart from the introduction of experimental data, more importantly, the intent of the present paper is to 
give insight on the driving force for aging of PC far below $T_{g}$ and therefore to understand the overall dynamics of PC in the glassy state.

To achieve this, all the PALS aging data including those from Ref. 14 are fitted by means of the Struik model. The equilibrium relaxation times that result from the fitting procedure are used to examine the nature of the aging process. In this framework, dielectric data are also presented to rationalize the various processes that govern physical aging.

\section{EXPERIMENT}

\section{A. Materials and sample preparation}

Polycarbonate (PC) from General Electric (Lexan 161) was used for all the experiments. Details on the sample geometry are given in Ref. 14. Before starting the aging experiments, PC samples were thermally rejuvenated at about $170^{\circ} \mathrm{C}$ and cooled down in ice. A large number of "fresh" samples were subjected to aging at 50 and $65^{\circ} \mathrm{C}$, and these were used for the PALS experiments (performed at room temperature) after different aging times.

\section{B. PALS experiments}

PALS measurements were performed at room temperature on all the above-mentioned samples, by exposing them to radioactive ${ }^{22} \mathrm{NaCl}$ with an activity of $10 \mu \mathrm{C}$ sandwiched between two Kapton foils in order to avoid direct contact of the samples with the radioactive source. Details on the experimental setup are given in Ref. 14. $2 \times 10^{6}$ counts were collected for each spectrum, which corresponds to an irradiation time of about $8 \mathrm{~h}$. As clearly seen in Fig. 2 of Ref. 34, where exactly the same source of this study was used, this irradiation time is short enough to avoid any charging effects, provided the a "fresh" sample is employed for each experiment as was done here.

The spectrum is analyzed using the program POSITRONFIT, which describes the spectrum as a convolution of the instrument resolution function and a finite number of negative exponentials plus the background as described by the following equation: ${ }^{36}$

$$
y(t)=R(t) \otimes\left(N \sum_{i=1}^{n} I_{i} \lambda_{i} \exp \left(-\lambda_{i} t\right)+B\right) .
$$

Here $R(t)$ is the resolution function of the system, $\lambda_{i}$ is the inverse of the annihilation time (annihilation rate), and $B$ is the background signal. All spectra were resolved in three lifetime components: a short one of 125 ps related to $p$-Ps annihilation, an intermediate one of $400 \mathrm{ps}$ related to free positron annihilation, and a long component $\left(\tau_{o-\mathrm{Ps}}\right)$ related to $o$-Ps annihilation.

\section{Dielectric experiments}

Dielectric experiments were performed using a highprecision dielectric analyzer (ALPHA analyzer, Novocontrol) in combination with a Novocontrol Quatro temperature system providing control of the sample temperature with an accuracy better than $\pm 50 \mathrm{mK}$. Measurements were per- formed over a wide frequency range $\left(10^{-1}-10^{7} \mathrm{~Hz}\right)$. Temperature-dependent measurements were carried out isothermally in steps of $5^{\circ} \mathrm{C}$ during cooling from 190 to $-140{ }^{\circ} \mathrm{C}$.

\section{RESULTS}

In our previous paper ${ }^{14}$ on the same PC, we already discussed the free volume relaxation after quenching from $170^{\circ} \mathrm{C}$ at 25,100 , and $128^{\circ} \mathrm{C}$ for aging times up to few thousands hours. The $o$-Ps lifetime does not show any variation with aging time, while its intensity clearly decays as aging proceeds. This implies that the cavity size is constant in all aging conditions while the number of cavities decreases. Analogous results were found for aging experiments through PALS at 50 and $65^{\circ} \mathrm{C}$. Figure 1 shows the relaxation of the excess free volume $v_{f 0}-v_{f \infty}=\Delta v_{f}$ as calculated from PALS data using Eqs. (4) and (5) and assuming $A$ $=0.023 \AA^{-3}$ as derived from literature data. ${ }^{37}$ Also, the values of $v_{f \infty o}$, the equilibrium free volume for each aging temperature, we used were obtained from free volume data in the available literature. ${ }^{37}$

\section{A. Implications of the Kauzmann paradox}

In extrapolating the excess free volume from data in the supercooled state, it was implicitly assumed that the equilibrium free volume is a linear function of temperature, even at temperatures far below $T_{g}$. However, the assumption that the equilibrium properties can be extrapolated from the rubbery state also gives rise to the so-called Kauzmann paradox. ${ }^{38}$ In this paradox it is noted that by extrapolation the heat capacity and the coefficient of thermal expansion of a supercooled liquid show a higher value than those of the crystal. This would lead to the paradoxical conclusion of the existence of a liquid phase below a certain temperature called the Kauzmann temperature $\left(T_{K}\right)$, with a smaller entropy and specific volume than the corresponding crystal. This idea led to the concept of an "ideal glassy state" that is normally not reached for kinetic reasons. ${ }^{39}$ However, some authors have objected to this view, showing for some particular glass formers that it is possible to attain a glassy state with smaller entropy than that of the crystal. ${ }^{40}$

In our case, we have extrapolated entropy data taken from the literature ${ }^{41}$ for supercooled PC and compared with the entropy of the crystalline PC. As it is possible to see in Fig. 2 , the $T_{K}$ for PC is located at $290 \mathrm{~K}$, which is less than the lowest aging temperature we use. Therefore, our assumption that $v_{f \infty}$ is a linear function of temperature can be assumed to be correct, independent of the presence of an ideal glassy state below $T_{K}$. It is also worth noting that for PC the value $T_{K}$ is not comparable with the Vogel temperature $T_{\mathrm{V}}$ (about $370 \mathrm{~K})$. This is in contrast with recent observations for lowmolecular-weight inorganic glass formers, which possess a $T_{K}$ that strongly resembles $T_{\mathrm{V}} \cdot{ }^{42}$ This was explained by correlating the thermodynamic properties with the relaxation behavior through an energy landscape model. ${ }^{43}$ However, this 


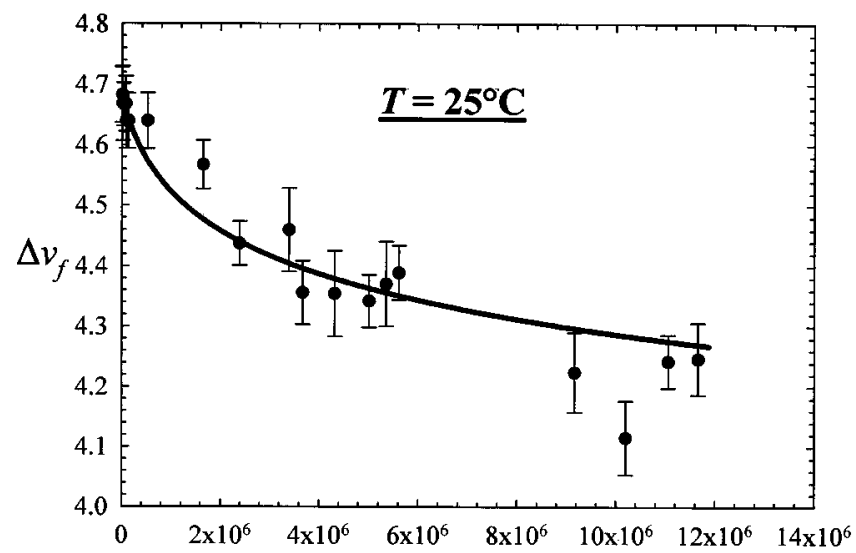

(a)

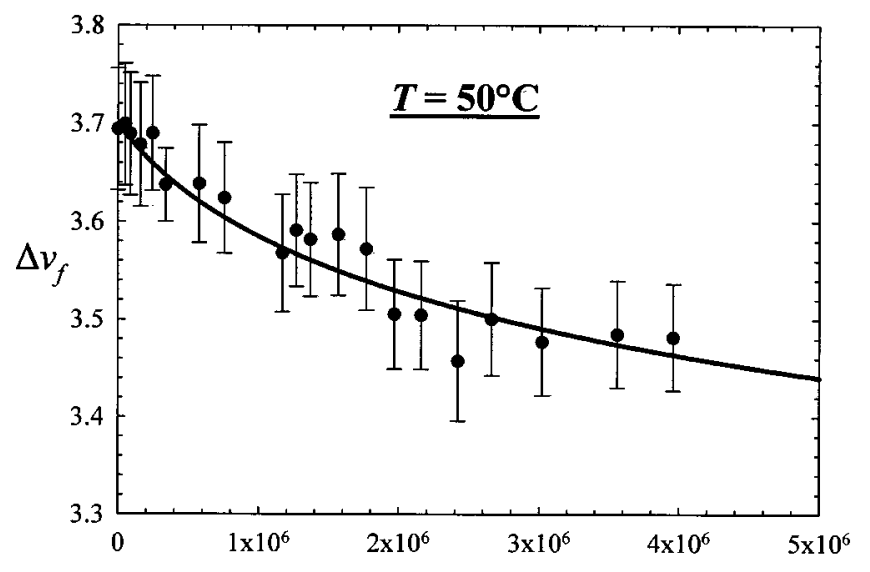

(b)
Time (s)

Time (s)

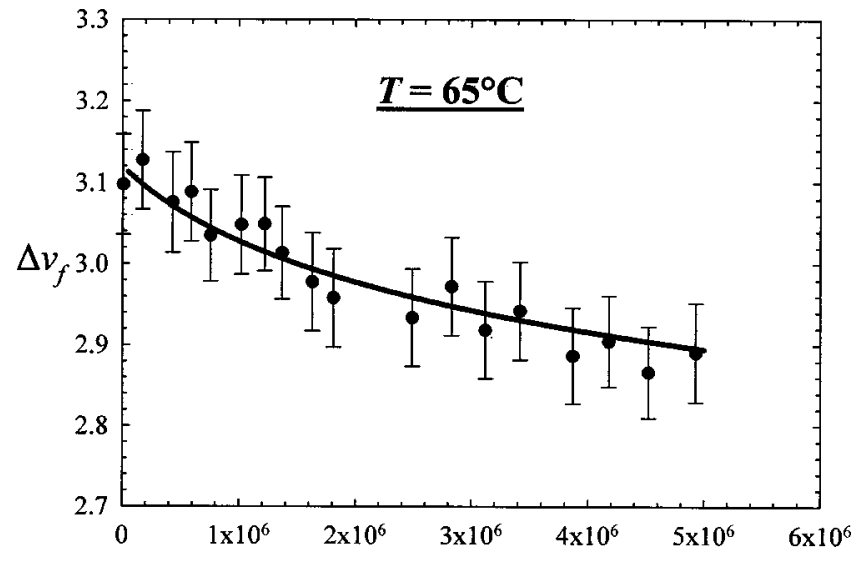

(c)

Time (s)

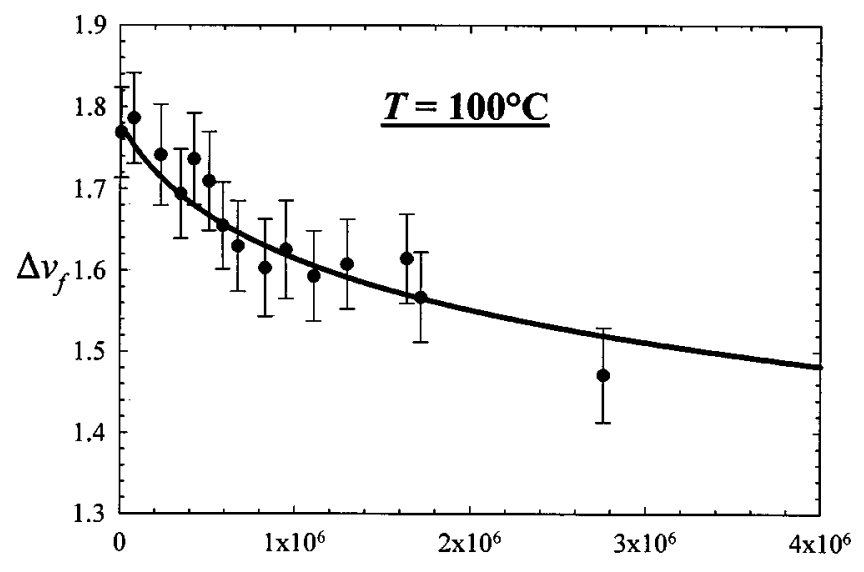

(d)

Time (s)

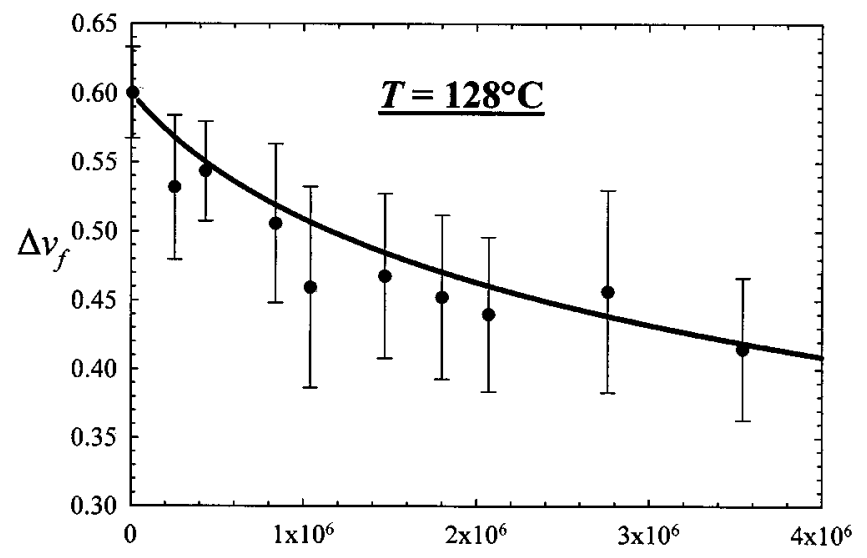

(e)

Time (s)

FIG. 1. Excess free volume development for samples aged at (a) $25^{\circ} \mathrm{C}$; (b) $50^{\circ} \mathrm{C}$; (c) $65{ }^{\circ} \mathrm{C}$; (d) $100{ }^{\circ} \mathrm{C}$; (e) $128^{\circ} \mathrm{C}$. Data at 25 , 100 , and $128^{\circ} \mathrm{C}$ are taken from Ref. 14 , adding error bars. The solid lines are from a fitting of the Struik model to the data.

correlation is not generally found for polymers and lowmolecular-weight organic glass formers. ${ }^{44-46}$ For example, for PC a low thermodynamic fragility, expressed by the ratio of the heat capacities in the liquid and in the glassy state, which corresponds to a relatively low $T_{K}$, is accompanied by a high dynamic fragility that in turns leads to a relatively high $T_{\mathrm{V}}$.

\section{B. Application of the Struik model}

The excess free volume relaxation patterns were modeled applying Eqs. (2) and (3) of the Struik model. In particular, the combination of the two equations gives

$$
\frac{d \Delta v_{f}}{d t}=-\frac{\Delta v_{f}}{\tau_{\infty} \exp \left(-\gamma \Delta v_{f}\right)},
$$




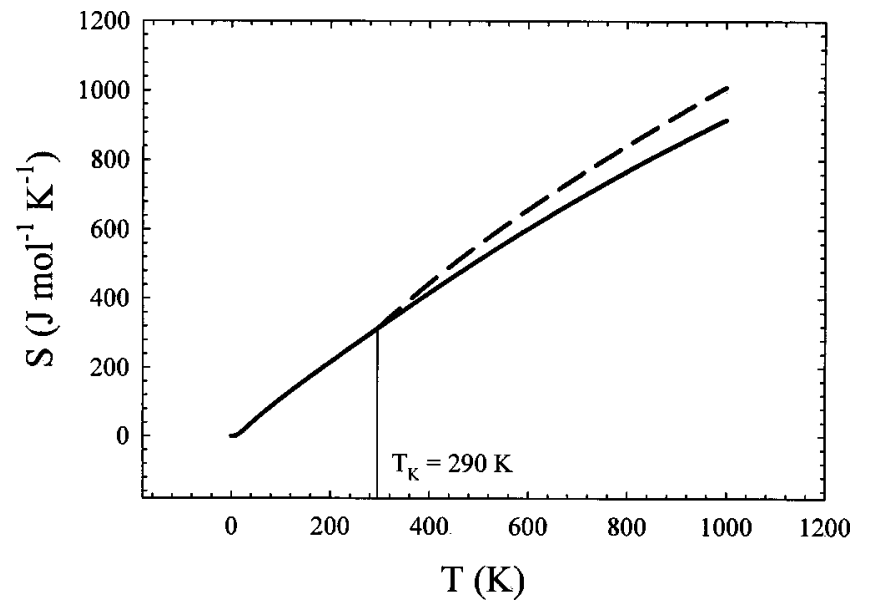

FIG. 2. Entropy vs temperature for PC amorphous (dashed line) and crystal (solid line) phases. While the crystal and amorphous phase data above $T_{g}$ are experimental, the entropy data for the amorphous phase below $T_{g}$ are extrapolated from $T_{g}$ to the Kauzmann temperature.

where $\tau_{\infty}=\tau_{0} \exp \left(E_{a} / k_{B} T\right)$ is the equilibrium value of the relaxation time.

Equation (7) was solved numerically and the values of the structural parameter $\gamma$ and of $\tau_{\infty}$ were obtained from the fitting to the experimental data. A nonlinear least square fitting procedure was employed for each curve. The solid lines in Fig. 1 are the fitting from the model. These results show that an excellent agreement between the experimental data points and the model can be obtained. It is important to note that a single value of $\gamma=8.45 \pm 0.05$ was obtained for all aging temperatures.

Figure 3 shows the equilibrium relaxation times $\tau_{\infty}$ obtained from the PALS data (open circles) with the relaxation data above $T_{g}$ for supercooled PC obtained through our dielectric spectroscopy data (open triangles). The relaxation time of the $\alpha$ process was obtained at each temperature from the maximum of $\varepsilon^{\prime \prime}$ in the isothermal frequency sweep plots. In addition, the relaxation times obtained by O'Connell and McKenna ${ }^{23}$ just below $T_{g}$ from creep experiments are also shown in the figure (open diamonds). The deviation from simple VFT behavior is clear. Examination of this figure reveals a remarkable continuity in the PALS equilibrium relaxation times for PC with those reported in literature ${ }^{23}$ and with our dielectric spectroscopy measurements. Furthermore, the equilibrium relaxation time at $128^{\circ} \mathrm{C}$ nearly overlaps with the relaxation time reported in Ref. 23. This indicates that the fitting of PALS data with the Struik model produces reasonable values of the equilibrium relaxation times at temperatures far below those normally investigated by dynamic techniques.

The equilibrium relaxation times below $T_{g}$ from PALS and from creep data ${ }^{23}$ were fitted with a fourth-order polynomial equation. This is shown in Fig. 3 by the solid line. Using the VFT fit from Ref. 23 above $T_{g}$ and the polynomial fit below $T_{g}$ allows us to calculate the activation energy shown in Fig. 4. This is done by taking the derivative of the logarithm of the relaxation time with the inverse absolute temperature. Note that this plot strongly resembles that ob-

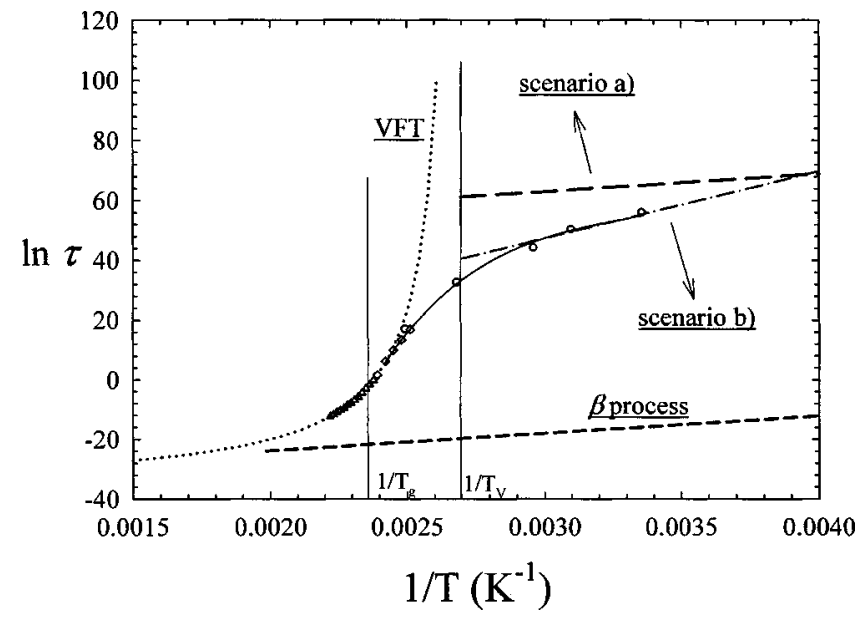

FIG. 3. Relaxation times for PC vs reciprocal temperature. Experimental data: $(\bigcirc)$ from the fitting of our PALS data of the Struik model; $(\diamond)$ relaxation data from Ref. 22; and $(\triangle)$ from dielectric data. Three regions can be distinguished: one above $T_{g}$, where the WLF-VFT holds; one between $T_{\mathrm{V}}$ and $T_{g}$, where the relaxation pattern starts to deviate from the WLF-VFT behavior; and a third one below $T_{\mathrm{V}}$, where the relaxation time either tends towards a $\beta$ activated process (dashed line), or follows an Arrhenius activated process with an activation energy larger than that of the $\beta$ process (dash-dotted line). The dotted line represents the VFT-WLF behavior. The short-dashed line represents the $\beta$ process.

tained by van Turnhout and Wübbenhorst ${ }^{47}$ by means the bivariate differential sampling of dielectric data for a number of low-molecular-weight glass formers. In that case, nonequilibrium activation energies are plotted at temperatures below $T_{g}$. However, in the same work, the effect of physical aging on the nonequilibrium activation energy was also investigated, showing only a slight upwards shift of the activation energy, while retaining the general shape of the curve.

\section{DISCUSSION}

In the preceding section, we obtained the relaxation time and the activation energy map in a wide temperature range for PC by employing relaxation data from dielectric spec-

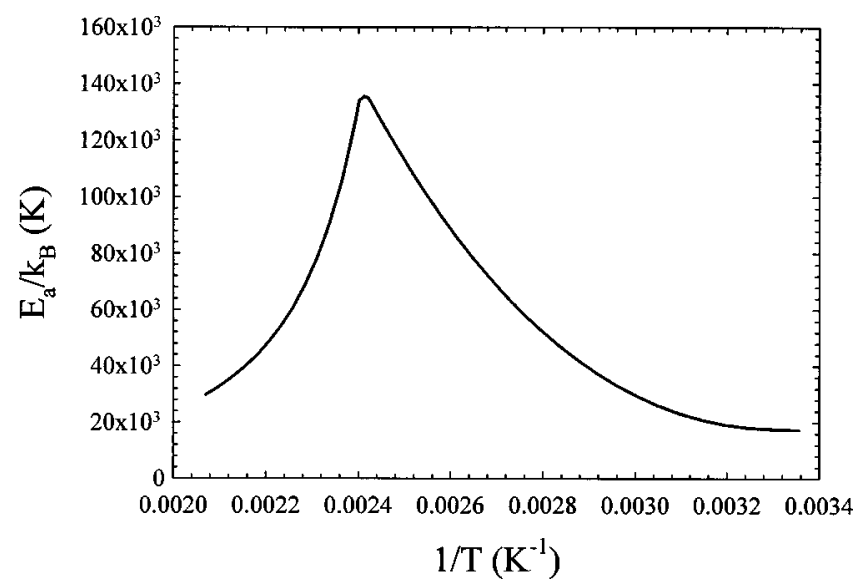

FIG. 4. Activation energy curve derived from the relaxation time behavior through the equation $d \ln \tau / d(1 / T)=E_{a} / k$. 
troscopy, literature data ${ }^{23}$ for temperatures just below $T_{g}$, and the relaxation times obtained after fitting of our experimental PALS data by means of the Struik model. If we analyze the data in terms of activation energy, we observe that the strong increase of the activation energy described by the VFT equation at high temperature is no longer present at temperatures below $T_{g}$. On the other hand, the activation energy initially seems to stabilize at a fixed value just below $T_{g}$ and starts even to decrease upon further temperature reduction. From the analysis of Fig. 4, a rather low value of the activation energy is obtained at 25,50 , and $65^{\circ} \mathrm{C}$ at about $E_{a} / k_{B}=20000 \mathrm{~K}$.

The activation energy described by the WLF fit of Mercier and Groeninckx ${ }^{48}$ for supercooled PC above $T_{g}$ and from the Arrhenius fit of the aging experiments of O'Connell and McKenna ${ }^{23}$ just below $T_{g}$, which reported an activation energy of $E_{a} / k_{B}=115230 \mathrm{~K}$, results in a much higher value. The trend of the relaxation time below $T_{g}$ suggests an influence that increases with decreasing temperature of the $\beta$ process, related to the flipping of the aromatic ring. ${ }^{49,50}$ However, it has been recently demonstrated that the $\beta$ process of PC is due to a cooperative motion that includes several repeat units. ${ }^{51}$ The activation of this process is reported to be about $E_{a} / k_{B}=6000 \mathrm{~K}$ from dielectric measurements. ${ }^{13}$

To explain these phenomena, we would like to propose two possible scenarios that will be elaborated below:

(a) The activation energy will decrease further for aging experiments at even lower temperatures and will approach the value of the $\beta$ process.

(b) The activation energy of the aging process assumes a constant value that is higher than the one of the $\beta$ process due to cooperativity.

\section{A. Activation energy tending to the $\boldsymbol{\beta}$ process}

The first scenario proposes that the relaxation processes are governed by the $\beta$ process for temperatures far below $T_{g}$. In particular, we can distinguish three different regimes in the relaxation pattern of PC as sketched in Figs. 3 and 4: (i) a VFT-WLF regime at temperatures above $T_{g}$, where the long-range relaxation related to the $\alpha$ process is dominant and controls the whole motion in the system; (ii) an intermediate regime just below $T_{g}$, where the long-range relaxation becomes more damped and the relaxation pattern deviates from the VFT-WLF behavior, due to the increasing contribution of the $\beta$ process, and which for a small temperature range resembles an Arrhenius behavior as described by O'Connell and McKenna ${ }^{23}$ and by other authors for other glass formers; ${ }^{20,22,24-26}$ (iii) a low-temperature regime where the $\alpha$ process starts to be heavily damped and the $\beta$ process dominates the dynamics of PC.

Due to the fact that the activation energy for our lowest aging temperatures does not reach the value of the activation energy of the $\beta$ process, we can assume that the $\alpha$ process still plays a minor role in the dynamics of PC, despite the fact that the mean relaxation time has already diverged to infinity as predicted by the VFT equation. A possible explanation is that the fast modes of the $\alpha$ process arising from the heterogeneity of the glassy state ${ }^{12}$ are still active even at

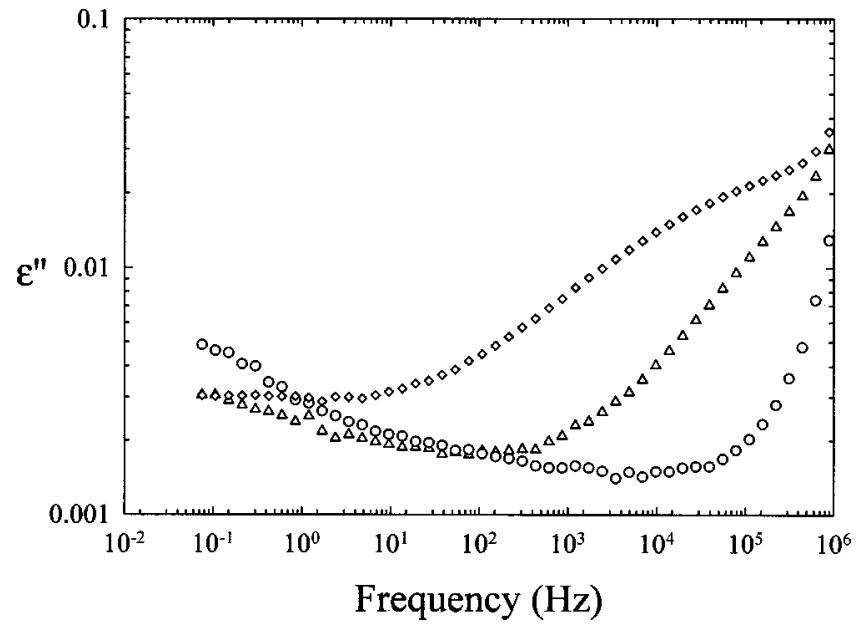

FIG. 5. Dielectric loss frequency response at $(\diamond)-20^{\circ} \mathrm{C} ;(\triangle)$ $25^{\circ} \mathrm{C}$; and $(\bigcirc) 100^{\circ} \mathrm{C}$. For clarity, data at the other aging temperatures are not shown.

temperatures below $T_{\mathrm{V}}$. This hypothesis is supported by the dielectric response of $\mathrm{PC}$ at our aging temperatures, shown in Fig. 5, which clearly exhibits a high-frequency tail attributable to the $\alpha$ process, even at $25^{\circ} \mathrm{C}$. The high-frequency tail eventually fades away, at least in the frequency window of our experiments, at $-20^{\circ} \mathrm{C}$. Not surprisingly, from the analysis of Fig. 3, this temperature approximately coincides with the temperature at which the $\beta$ process totally governs the aging process, while even the high-frequency tail of the $\alpha$ process has become too slow (frequency smaller than $0.1 \mathrm{~Hz}$ ) to produce effects on a macroscopic scale, at least on the time scale of our aging experiments.

The prominent role of the $\beta$ process in regulating physical aging deep in the glassy state is supported by the creep experiments of Struik, ${ }^{17}$ who showed that the aging process progressively vanishes below $T_{\beta}$ (defined as the temperature at which the dielectric or mechanical loss $\beta$ peak has a maximum at $1 \mathrm{~Hz}$ ). Moreover, the shift rate $\mu=-\log a_{T} / \log t_{a g}$, i.e., the ratio of the logarithm of the horizontal shift of the creep experiments and of the aging time, is approximately 1 between $T_{\beta}$ and $T_{g}$ for the most common amorphous glassy polymers. Of course, the $\beta$ process will be still active at temperatures lower than $T_{\beta}$, but, evidently, its frequency progressively becomes too small to produce significant macroscopic effects. Additional support is also given by the potential energy landscape view for supercooled liquids by Stillinger. ${ }^{52}$ As temperature decreases, the configuration point has to overcome increasingly higher barriers to pass from one basin to another, and only the lowest barriers related to $\alpha$ rearrangements (the fast processes) and the low barriers related to the elementary transitions of the $\beta$ process can be overcome. Ultimately, at temperatures below $T_{\beta}$, these barriers also become too high for the available thermal energy, and the system is totally frozen. Moreover, it has to be mentioned that the dominant role of the $\beta$ process in regulating the relaxation processes far below $T_{g}$ was recently shown for amorphous polyethylene naphthalate (PEN). ${ }^{53} \mathrm{In}$ particular, uniaxial stress tension relaxation experiments displayed a power law dependence of the relaxation modulus on 
the relaxation time, which is typically related to the contribution of the $\beta$ process to the overall relaxation response. As the temperature and/or the time is increased the dependence starts to be exponential, indicating that the $\alpha$ process starts to play a significant role in determining the relaxation response. Finally, the possibility that at very low temperatures the $\beta$ process completely governs the physical aging process is compatible with the highly cooperative nature of this process as previously mentioned. ${ }^{51}$

\section{B. Activation energy higher than that of the $\boldsymbol{\beta}$ process}

A second possible scenario is based on the idea that in order to have an effect on the macroscopic properties, the local segmental motion related to the $\beta$ process requires a certain degree of cooperativity. In contrast to the previous case, this scenario would be valid independent of the $\alpha$ process in producing macroscopic effects, and does not require the hypothesis of a heterogeneous nature of the glassy state. In fact, this concept is rejected by some authors who provide evidence for a homogeneous scenario. ${ }^{54}$ In this case, $T_{\mathrm{V}}$ can be perceived as a real singularity where all the relaxations related to the $\alpha$ process become frozen. Contrary to the previous scenario, the fact that activation energy assumes a value higher than that of the $\beta$ process at our lowest aging temperatures is explained by supposing that only a specific sequence of coupled $\beta$ events can produce an effect on the macroscopic thermodynamic properties. If we fit the relaxation time for our three lowest aging temperatures $(25,50$, and $65^{\circ} \mathrm{C}$ ) with a linear function, we find an activation energy of $E_{a} / k=22500 \mathrm{~K}$, which is almost four times larger than that of the $\beta$ process but still about one order of magnitude smaller than that of the $\alpha$ process. This situation is sketched in Fig. 4 with a dash-dotted line. From a potential energy barrier point of view, this scenario requires the sequential transition of a number of $\beta$ relaxation barriers in order to produce a variation of the macroscopic properties.

\section{Length and time scale of the aging process}

In both of the two scenarios described previously, a prominent role of the $\beta$ process in influencing the aging process far below $T_{g}$ is apparent. At room temperature, the typical time for relaxation is of the order of $10^{7} \mathrm{~s}$, while that for a single $\beta$ rearrangement takes place in $10^{-7} \mathrm{~s}$. This large difference can be interpreted using several theories of aging presented in the past by different authors that are all based on a free volume diffusion mechanism for aging of polymers.

According to Curro et al. ${ }^{55}$ who modeled the volume recovery of poly(vinyl acetate), the internal length scale for diffusion could be attributed to either an internal annihilation of vacancies or to a redistribution of free volume in an inhomogeneous structure. Perez ${ }^{56}$ modeled the volume and enthalpy of poly(vinyl acetate) according to the migration and annihilation of defects of different signs corresponding to sites with positive and negative density fluctuation. Recently, McCaig et al. ${ }^{57,58}$ investigated the variation of the permeation of oxygen with time on samples of polyarylate deep in the glassy state with different thicknesses. Their results showed a thickness-dependent aging mechanism, which they attributed to diffusion towards the surface of the sample. Similar results were obtained by Dorkenoo and Pfromm for poly[1-(trimethylsilyl)-1-propyne] (PTMSP). ${ }^{59}$ Since the sample thickness starts to influence the aging process at a thickness of few microns, a typical length scale for diffusion of that order of magnitude may be appropriate. In our case, if we assume a diffusion mechanism for aging driven by the $\beta$ process, the following equation can be applied:

$$
\tau_{a g}=\xi \tau_{\beta}\left(\frac{l}{b}\right)^{2}
$$

where $\tau_{a g}$ and $\tau_{\beta}$ are, respectively, the relaxation time for the aging process and that for the $\beta$ process, $l$ and $b$ are the typical length scales for the two processes and $\xi$ is a coupling parameter, which we have introduced to take into account the coupling between the $\beta$ relaxation and the free-volume changes during physical aging.

If we employ a typical length scale of say $10 \mu \mathrm{m}$ as suggested by McCaig and Paul ${ }^{57}$ for a polymer that is deep in the glassy state and a length scale for the $\beta$ process of 10 $\AA$, we obtain $\xi=10^{6}$. The high value of $\xi$ suggests that, although the $\beta$ process dominates the physical aging, the coupling between the two processes is very weak, i.e., the efficiency of the $\beta$ relaxation in producing changes in the macroscopic properties of the polymer is very low. In other words, while the vast majority of the $\beta$ events is not effective in producing a macroscopic relaxation of the system, a very small fraction cooperates to give an effect in the evolution of the macroscopic properties.

Therefore this may explain the vastly different time scale for aging and $\beta$ dynamics, which is in accordance with the observed lack of coupling between the $\beta$ peak and external pressure $^{60}$ as observed for a Johari-Goldstein type of $\beta$ relaxation. In the present case there is some cooperativity, even for the single $\beta$ event, ${ }^{51,61}$ which may influence this result to some extent although a strong coupling of the $\beta$ time scale to the external pressure would not be expected. Also note that, in relation to the second scenario, the product $\xi \tau_{\beta}=10^{-1} \mathrm{~s}$ would be simply the characteristic time scale for a cooperative relaxation involving a number of coupled $\beta$ relaxations.

\section{CONCLUSIONS}

Aging of PC was studied by monitoring the free volume relaxation deep in the glassy state after quenching from above $T_{g}$, by means of PALS, to provide more insight about PC dynamics below $T_{g}$, starting from recent observations that just below this temperature, deviations from the VFTWLF behavior occur. The PALS data were examined using a model for aging proposed by Struik, which gives an excellent fitting to our experimental data. The temperature dependence of the relaxation time was obtained using our dielectric data above $T_{g}$, literature data just below $T_{g}$, and our data far below $T_{g}$. The analysis of the relaxation time trend suggests two possible scenarios: (a) The lower the temperature, the more the aging dynamics of PC is dominated by the $\beta$ process, while the $\alpha$ process, which relates to cooperative 
motion on a larger scale, is gradually frozen, and only remains present via its high-frequency modes. (b) The aging process is observable as a change of the macroscopic properties, thanks to a process that involves the cooperation of a number $\beta$ events, resulting in an activation energy about four times higher than that of the $\beta$ process. The values of the typical relaxation times for the aging process and the $\beta$ process were applied to a diffusion mechanism for aging and a coupling parameter was introduced, which showed a very low efficiency of the $\beta$ relaxation process in producing a measurable variation of the macroscopic properties.

\section{ACKNOWLEDGMENTS}

The work of D.C. and S.J.P. forms part of the research program of the Dutch Polymer Institute (DPI). This work is carried out under DPI Project No. 285: "Influence of Applied Stress and Physical Ageing on Diffusion and Solubility of Oxygen and Anti-Oxidants."
${ }^{1}$ J. D. Ferry, Viscoelastic Properties of Polymers, 3rd ed. (Wiley, New York, 1980).

${ }^{2}$ H. Vogel, Phys. Z. 22, 645 (1921).

${ }^{3}$ G. Tammann and G. Hesse, Z. Anorg. Allg. Chem. 156, 245 (1926).

${ }^{4}$ G. S. Fulcher, J. Am. Ceram. Soc. 8, 339 (1925).

${ }^{5}$ M. L. Williams, R. F. Landel, and J. D. Ferry, J. Am. Chem. Soc. 77, 3701 (1955).

${ }^{6}$ C. A. Angell, In Relaxations of Complex Systems, edited by K. L. Ngai and G. B. Wright (National Technical Information Service, U.S. Department of Commerce, Springfield, VA 1985).

${ }^{7}$ C. A. Angell, K. L. Ngai, G. B. McKenna, P. F. McMillan, and S. W. Martin, J. Appl. Phys. 88, 3113 (2000).

${ }^{8}$ G. Williams and D. C. Watts, Trans. Faraday Soc. 66, 80 (1970).

${ }^{9}$ R. Bühmer, K. L. Ngai, C. A. Angell, and D. J. Plazek, J. Chem. Phys. 99, 4201 (1993).

${ }^{10}$ T. A. Vilgis, Phys. Rev. B 47, 2882 (1993).

${ }^{11}$ K. L. Ngai, A. Sokolov, and W. Steffen, J. Chem. Phys. 107, 5268 (1997).

${ }^{12}$ H. Sillescu, R. Böhmer, G. Diezemann, and G. Hinze, J. NonCryst. Solids 307, 16 (2002).

${ }^{13}$ N. G. McCrum, B. E. Read, and G. Williams, Anelastic and Dielectric Effects in Polymeric Solids (Dover, New York, 1991).

${ }^{14}$ D. Cangialosi, H. Schut, A. van Veen, and S. J. Picken, Macromolecules 36, 142 (2003).

${ }^{15}$ R. Wimberger-Friedl and J. G. de Bruin, Macromolecules 29, 4992 (1996).

${ }^{16}$ C. G. Robertson and G. L. Wilkes, Macromolecules 33, 3954 (2000).

${ }^{17}$ L. C. E. Struik, Physical Aging in Amorphous Glassy Polymers and Other Materials (Elsevier Science, Amsterdam, 1978).

${ }^{18}$ A. K. Doolittle, J. Appl. Phys. 22, 1471 (1951).

${ }^{19}$ G. Adam and J. H. Gibbs, J. Chem. Phys. 43, 139 (1965).

${ }^{20}$ R. Nozaki and S. Mashimo, J. Chem. Phys. 87, 2271 (1987).

${ }^{21}$ E. A. Di Marzio and A. J. M. Yang, J. Res. Natl. Inst. Stand. Technol. 102, 135 (1997).

${ }^{22}$ S. L. Simon, J. W. Sobieski, and D. J. Plazek, Polymer 42, 2555 (2001).

${ }^{23}$ P. A. O'Connell and G. B. McKenna, J. Chem. Phys. 110, 11054 (1999).

${ }^{24}$ A. Alegria, E. Guerrica-Echevarria, I. Telleria, and J. Colmenero, Phys. Rev. B 47, 14857 (1993).

${ }^{25}$ C. T. Thurau and M. D. Ediger, J. Chem. Phys. 118, 1996 (2003).

${ }^{26}$ J. K. Kruger, T. Britz, J. Baller, W. Possart, and H. Neurohr, Phys. Rev. Lett. 89, 285701 (2002).

${ }^{27}$ L. C. E. Struik, Physical Aging in Amorphous Glassy Polymers and Other Materials (Elsevier Science, Amsterdam, 1978), pp. 42-46.

${ }^{28}$ O. E. Mogensen, Positron Annihilation in Chemistry (SpringerVerlag, Berlin, 1995).

${ }^{29}$ M. Eldrup, D. Lightbody, and J. N. Sherwood, Chem. Phys. 63, 51 (1981).

${ }^{30}$ Y. Kobayashi, W. Zheng, E. F. Meyer, J. McGervey, A. Jamieson, and R. Simha, Macromolecules 22, 2302 (1989).

${ }^{31}$ A. J. Hill, I. M. Katz, and P. L. Jones, Polym. Eng. Sci. 30, 762 (1990).

${ }^{32}$ A. J. Hill and C. M. Agrawal, J. Mater. Sci. 25, 5036 (1990); A. J. Hill, K. J. Heater, and C. M. Agrawal, J. Polym. Sci., Part B: Polym. Phys. 28, 387 (1990); A. J. Hill and P. L. Jones, Structure Relaxation and Physical Aging of Glassy Polymers, edited by R. J. Roe, J. M. Torkelson, and J. M. O'Reilly, Mater. Res. Soc. Symp. Proc. 215 (Materials Research Society, Pittsburgh, PA, 1991), p. 175; K. J. Heater and P. L. Jones, ibid. 215, p. 206 (1991); W. J. Davies and R. A. Pethrick, Eur. Polym. J. 34, 1747 (1998).

${ }^{33}$ J. M. Hutchinson, Prog. Polym. Sci. 20, 703 (1995).

${ }^{34}$ D. Cangialosi, H. Schut, M. Wübbenhorst, J. van Turnhout, and A. van Veen, Radiat. Phys. Chem. 68, 507 (2003).

${ }^{35}$ T. Hirade, F. H. J. Maurer, and M. Eldrup, Radiat. Phys. Chem. 58, 465 (2000); R. A. Naslud, Mater. Sci. Forum 175, 739 (1995); X. S. Li and M. C. Boyce, J. Polym. Sci., Part B: Polym. Phys. 31, 869 (1993); J. E. Kluin, Z. Yu, S. Vleeshouwers, J. D. McGervey, A. M. Jamieson, and R. Simha, Macromolecules 25, 5089 (1992).

${ }^{36}$ P. Kirkegaard, M. Eldrup, O. E. Mogensen, and N. J. Pedersen, Comput. Phys. Commun. 23, 307 (1981).

${ }^{37}$ K. Hagiwara, T. Ougizawa, T. Inoue, K. Hirata, and Y. Kobayashi, Radiat. Phys. Chem. 58, 525 (2000).

${ }^{38}$ W. Kauzmann, Chem. Rev. (Washington, D.C.) 43, 219 (1948).

${ }^{39}$ J. Jäckle, Rep. Prog. Phys. 49, 171 (1986).

${ }^{40}$ F. H. Stillinger, P. G. Debenedetti, and T. M. Truskett, J. Phys. Chem. B 105, 11809 (2001).

${ }^{41}$ ATHAS Data Bank update 1989.

${ }^{42}$ C. A. Angell, J. Res. Natl. Inst. Stand. Technol. 102, 171 (1997).

${ }^{43}$ C. A. Angell, Science 267, 1924 (1995).

${ }^{44}$ C. M. Roland, P. G. Santangelo, and K. L. Ngai, J. Chem. Phys. 111, 5593 (1999).

${ }^{45}$ D. Huang and G. B. McKenna, J. Chem. Phys. 114, 5621 (2001).

${ }^{46}$ H. Tanaka, Phys. Rev. Lett. 90, 55701 (2003).

${ }^{47}$ J. van Turnhout and M. Wübbenhorst, J. Non-Cryst. Solids 305, 50 (2002).

${ }^{48}$ J. P. Mercier and G. Groeninckx, Rheol. Acta 8, 510 (1969).

${ }^{49}$ P. M. Henrichs and V. A. Nicely, Macromolecules 24, 2506 (1991). 
${ }^{50}$ A. K. Roy, A. A. Jones, and P. T. Inglefield, Macromolecules 19, 1356 (1986).

${ }^{51}$ J. Y. Jho and A. F. Yee, Macromolecules 24, 1905 (1991).

${ }^{52}$ F. H. Stillinger, Science 267, 1935 (1995).

${ }^{53}$ M. Cerrada and G. B. McKenna, Macromolecules 33, 3065 (2000).

${ }^{54}$ A. Arbe, J. Colmenero, M. Monkenbusch, and D. Richter, Phys. Rev. Lett. 81, 590 (1998).

${ }^{55}$ J. G. Curro, R. R. Lagasse, and R. Simha, Macromolecules 15, 1621 (1982).
${ }^{56}$ J. Perez, Polymer 29, 483 (1987).

${ }^{57}$ M. S. McCaig and D. R. Paul, Polymer 41, 629 (2000).

${ }^{58}$ M. S. McCaig, D. R. Paul, and J. W. Barlow, Polymer 41, 639 (2000).

${ }^{59}$ K. D. Dorkenoo and P. H. Pfromm, Macromolecules 33, 3747 (2000).

${ }^{60}$ S. Hensel-Bielowka, M. Paluch, J. Ziolo, and C. M. Roland, J. Phys. Chem. B 106, 12459 (2002).

${ }^{61}$ K. L. Ngai, R. W. Rendell, and A. F. Yee, Macromolecules 21, 3396 (1988). 